Article

\title{
Atmospheric Effects on OFDM Wireless Links Operating in the Millimeter Wave Regime
}

\author{
Yosef Golovachev ${ }^{1, *} \mathbb{D}$, Gad A. Pinhasi ${ }^{2, *}$ and Yosef Pinhasi ${ }^{1, *}$ \\ 1 Department of Electrical and Electronic Engineering, Ariel University, Ariel 98603, Israel \\ 2 Department of Chemical Engineering, Ariel University, Ariel 98603, Israel \\ * Correspondence: yosi_habad@yahoo.com (Y.G.); gadip@ariel.ac.il (G.A.P.); yosip@ariel.ac.il (Y.P.)
}

Received: 6 September 2020; Accepted: 27 September 2020; Published: 29 September 2020

check for updates

\begin{abstract}
The development of millimeter wave communication links and the allocation of bands within the Extremely High Frequency (EHF) range for the next generation cellular network present significant challenges due to the unique propagation effects emerging in this regime of frequencies. This includes susceptibility to amplitude and phase distortions caused by weather conditions. In the current paper, the widely used Orthogonal Division Frequency Multiplexing (OFDM) transmission scheme is tested for resilience against weather-induced attenuation and phase shifts, focusing on the effect of rainfall rates. Operating frequency bands, channel bandwidth, and other modulation parameters were selected according to the 3rd Generation Partnership Project (3GPP) Technical Specification. The performance and the quality of the wireless link is analyzed via constellation diagram and BER (Bit Error Rate) performance chart. Simulation results indicate that OFDM channel performance can be significantly improved by consideration of the local atmospheric conditions while decoding the information by the receiver demodulator. It is also demonstrated that monitoring the weather conditions and employing a corresponding phase compensation assist in the correction of signal distortions caused by the atmospheric dispersion, and consequently leads to a lower bit error rate.
\end{abstract}

Keywords: MMW; OFDM; 5G; 3GPP; atmospheric radio propagation

\section{Introduction}

The rapid growing use of cellular services and the expanding line of products that are based on the wireless communications technologies have led to an increasing interest in the standardization of the 5th Generation (5G) mobile communication technology. The 5G technology is expected to use millimeter waves (MMW) to offer unprecedented spectrum and extremely high data rates to the wireless communications. The implementation of MMW technology has led to the recognition of the necessity of a consistent standardization of networks, channels, and modulation techniques that are relied on corresponding modeling efforts [1].

The US Federal Communications Commission (FCC) has stated their intention to establish the U.S. as leaders in the field of 5G [2]. One of the options considered for application was the allocation of bandwidth in the Extremely High Frequency (EHF) range, 30-300 GHz that can lead to the development of practical consumer applications of MMW technologies. The spectrum available for licensed use was set in three bands within the Ultra High Frequency (UHF) $850 \mathrm{MHz}, 2.4 \mathrm{GHz}$, as well as MMW in the $28 \mathrm{GHz}, 37 \mathrm{GHz}$, and $39 \mathrm{GHz}$ bands. Furthermore, the 64-71 GHz band was recently designated for unlicensed use, which increases the total amount of unlicensed spectrum to $14 \mathrm{GHz}$ as well as $600 \mathrm{MHz}$ in the $37 \mathrm{GHz}$ band for shared access.

Different modulation techniques have been proposed for usage in 5G systems in order to provide greater flexibility and thereby increase transmission efficiency and reliability. Currently, the orthogonal 
frequency-division multiplexing (OFDM) scheme is used in the $4 \mathrm{G}$ as a digital multi-carrier modulation method and is one of the popular techniques considered for the 5G cellular wireless communications [3].

The communication in the EHF range has many advantages; however, it is subjected to atmospheric propagation issues. In general, atmospheric effects are due to absorption and dispersion phenomena. In short wavelengths, the MMW propagation is significantly affected by weather conditions, such as air pressure, temperature, relative humidity, fog, and rain.

Almost all of the studies on the effect of atmospheric conditions on the performance of communication links are referring to the attenuation only [4]. The signal phase shift and time delay effects are usually ignored, although they can be significant in extreme conditions $[5,6]$.

Pinhasi et al. presented in their previous studies on MMW atmospheric propagation, theoretical and experimental results of absorptive and dispersive propagation effects and their influence on the signal strength and phase dispersion [7-11]. It was found that apart from attenuation, the dispersive effects like group delay should be considered in the design of MMW and THz radar and wireless communication links.

In the present work, the performance of an OFDM wireless communication link operating in the MMW regime is studied under different weather conditions such as humidity, fog, and rain. The atmospheric effects on the OFDM waveform were formulated theoretically and demonstrated by simulation model.

For this purpose, an atmospheric channel model for wireless communication was developed, combining our MMW propagation model with 5G Toolbox of MATLAB R2019b (MathWorks, Natick, MA, USA) [12]. MMW propagation model is applied to the transmitted baseband OFDM complex envelope and the performance of the wireless link is presented by using constellation diagram and BER (Bit Error Rate) performance chart. The present work is focused on the dispersive effects like group delay and phase shift and their effect on the OFDM communication link.

The current paper presents an integration of several issues as sub-models: the MMW propagation model (Section 2), the frequency dependent attenuation and phase dispersion factors for different weather conditions (Section 3), transmission of OFDM waveform in a medium (Section 4), technical specifications of the next generation cellular network and the simulation parameters (Section 5). Section 6 presents simulations for MMW communication scenarios. The results of the simulation are presented in the form of a constellation diagram and BER performance graph for different atmospheric conditions, focusing on rainfall rate effect (Section 7). The novel approach of predicting phase distortions due to the dispersion of the millimeter waves propagating in the atmospheric medium is presented in Section 8. It is shown that by employing an adaptive phase compensator, the bit error rate can be considerably reduced.

\section{Channel Model}

Besides modelling of the transmitter and receiver, analyzing the wireless link operating in the millimeter wave regime also requires accurate description of the medium. The transmitted signal $E_{T}(t)$ is propagating in the medium, which can be treated as a linear system via its impulse response $h(t)$. The received signal is the result of the convolution $E_{R}(t)=E_{T}(t) * h(t)$. In the presence of Additive White Gaussian Noise (AWGN) $n(t)$, the signal and noise obtained at the receiver's input and fed to the demodulator is the summation $E_{\text {in }}(t)=E_{R}(t)+n(t)$. The link is illustrated in a block diagram in Figure 1.

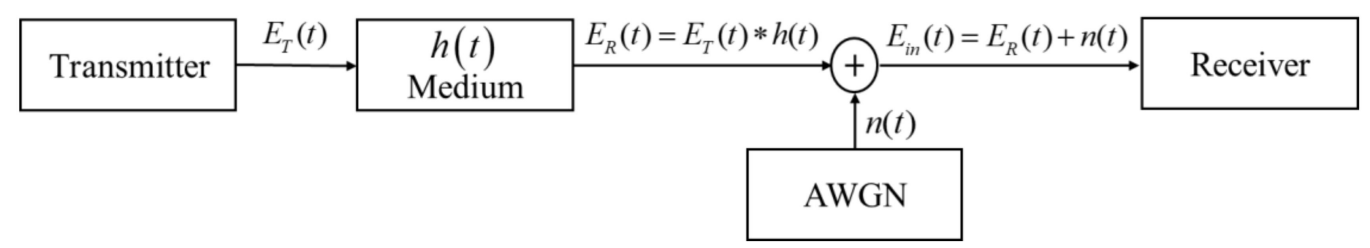

Figure 1. Block diagram of a wireless link operating in the millimeter wavelengths. 
The atmospheric medium is characterized as a linear system with a transfer function $H(j f)$ given in the frequency domain. In the time domain, the transmitted signal $E_{T}(t)$ is presented as a carrier wave at a frequency $f_{c}$ modulated by a wideband complex envelope $\widetilde{A}_{\text {in }}(t)$ [8]:

$$
E_{T}(t)=\operatorname{Re}\{\underbrace{[i(t)+j q(t)]}_{\widetilde{A}_{\text {in }}(t)} \cdot e^{j 2 \pi f_{c} t}\}=\frac{1}{2} \widetilde{A}_{\text {in }}(t) \cdot e^{+j 2 \pi f_{c} t}+\frac{1}{2} \widetilde{A}_{\text {in }}{ }^{*}(t) \cdot e^{-j 2 \pi f_{c} t}
$$

where $\widetilde{A}_{\text {in }}(t)=i(t)+j q(t)$ is a complex envelope, given in terms of the In-phase $i(t)$ and Quadrature $q(t)$ baseband components. A Fourier transformation of Equation (1) is carried out to present the transmitted signal in the frequency domain:

$$
\begin{gathered}
E_{T}(j f)=\mathcal{F}\left\{E_{T}(t)\right\} \equiv \int_{-\infty}^{+\infty} E_{T}(t) \cdot e^{-j 2 \pi f t} d t= \\
=\frac{1}{2} \widetilde{A}_{i n}\left[j\left(f-f_{c}\right)\right]+\frac{1}{2} \widetilde{A}_{\text {in }}{ }^{*}\left[-j\left(f+f_{c}\right)\right]
\end{gathered}
$$

where $\widetilde{A}_{\text {in }}(j f)=\mathcal{F}\left\{\widetilde{A}_{\text {in }}(t)\right\}$. Using the transfer function $H(j f)$ of the medium, the received signal can be now expressed in the frequency domain by:

$$
\begin{gathered}
E_{R}(j f)=E_{T}(j f) \cdot H(j f)= \\
=\frac{1}{2} \widetilde{A}_{\text {in }}\left[j\left(f-f_{c}\right)\right] \cdot H(j f)+\frac{1}{2} \widetilde{A}_{\text {in }}{ }^{*}\left[-j\left(f+f_{c}\right)\right] \cdot H(j f)
\end{gathered}
$$

Inverse Fourier transformation of Equation (3) results in the signal obtained at the receiver site:

$$
\begin{gathered}
E_{R}(t)=\int_{-\infty}^{+\infty} E_{R}(j f) e^{+j 2 \pi f t} d f= \\
=\operatorname{Re}\{\underbrace{\left.\int_{-\infty}^{\left.\int_{-\infty}^{+\infty} \widetilde{A}_{\text {in }}(j f) \cdot H\left[j\left(f+f_{c}\right)\right] \cdot e^{+j 2 \pi f t} d f\right\}}\right\} e^{j 2 \pi f_{c t} t}}_{\widetilde{A}_{\text {out }}(t)}\}
\end{gathered}
$$

The expression for the received complex envelope is identified in Equation (4) to be:

$$
\widetilde{A}_{\text {out }}(t)=\int_{-\infty}^{+\infty} \widetilde{A}_{\text {in }}(j f) \cdot H\left[j\left(f+f_{c}\right)\right] \cdot e^{+j 2 \pi f t} d f
$$

\section{Propagation Factors of the Atmosphere}

The millimeter wave propagation model (MPM) is used for calculation of the atmospheric frequency response taken into consideration of weather conditions. Contributions of dry air, water vapor, suspended water droplets (haze, fog, cloud), and rain are all addressed in the model. For completeness and clarity of notations, the model $[7,9]$ is hereby reviewed.

In general, the propagation of millimeter waves through the atmospheric medium along a distance $d$ is described by the transfer function:

$$
H(j f)=\sqrt{T_{F S}(d)} e^{-j \frac{2 \pi f}{c} \cdot n(f) \cdot d}
$$


where $n(f)$ is the refractive index of the atmospheric medium and $c$ is the speed of light in vacuum. $T_{F S}(d)$ is the free-space power transmission along a distance $d$ excluding the atmospheric transmittance:

$$
T_{F S}(d)=G_{R}\left(\frac{c}{2 \pi f_{c} d}\right)^{2} G_{T}
$$

where $G_{R}$ and $G_{T}$ are the directivity gains of the receiving and transmitting antennas, respectively. The refractive index $n(f)$ is a frequency dependent quantity that can be expressed in terms of dispersive complex refractivity $N(f)$ (given in ppm):

$$
n(f)=1+\underbrace{\left[N_{0}+N^{\prime}(f)-j N^{\prime \prime}(f)\right]}_{N(f)} \times 10^{6}
$$

where the nondispersive part $N_{0}$ is real and positive constant, $N^{\prime}(f)$ and $N^{\prime \prime}(f)$ are the frequency dependent real and imaginary refractivity terms, respectively. The millimeter wave propagation model (MPM) is used for calculation of these refractivity terms in the frequency domain [13,14].

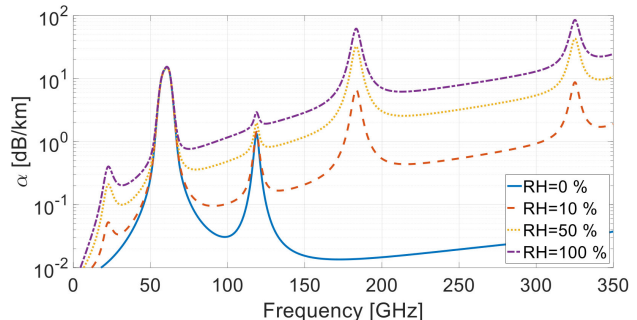

(a)

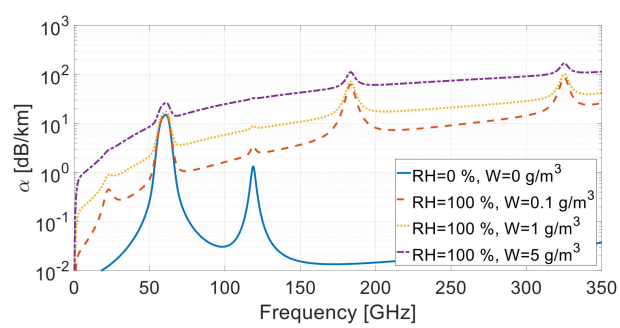

(c)

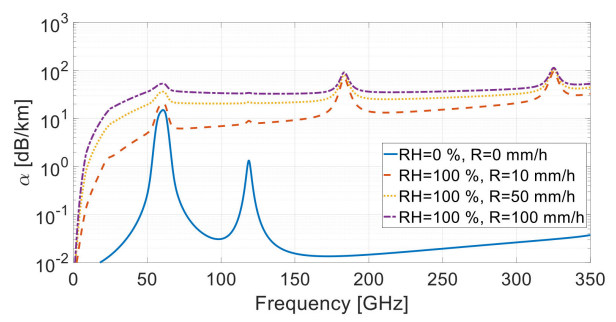

(e)

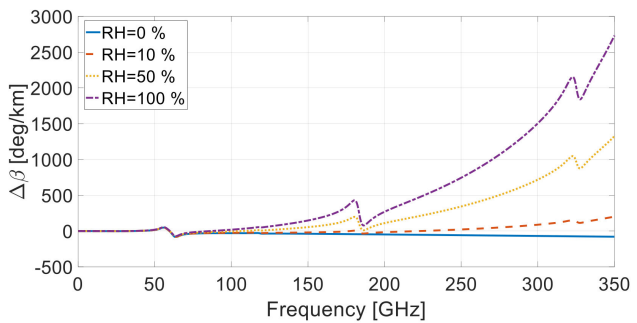

(b)

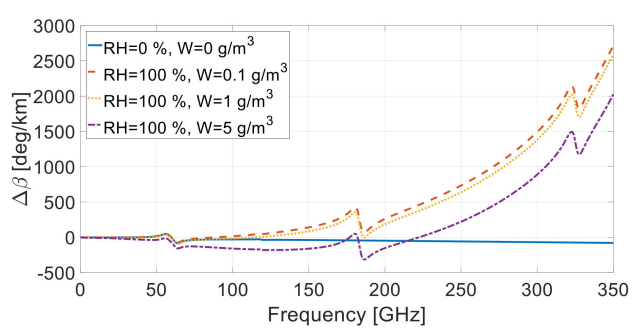

(d)

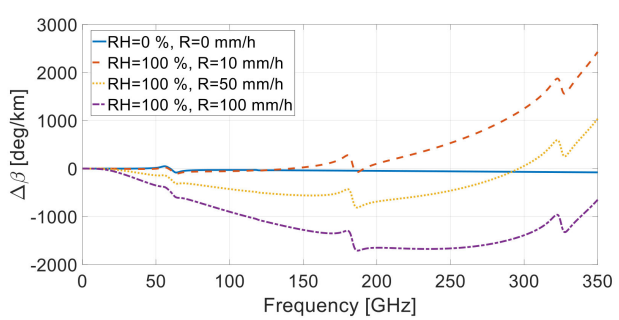

(f)

Figure 2. Attenuation factor $(\mathbf{a}, \mathbf{c}, \mathbf{e})$ and incremental phase dispersion factor $(\mathbf{b}, \mathbf{d}, \mathbf{f})$ in different weather conditions: (a,b) show the relative humidity $\mathrm{RH}[\%]$ effect $(\mathbf{c}, \mathbf{d})$ show the effect of suspended droplets via the liquid water content $\mathrm{W}\left[\mathrm{g} / \mathrm{m}^{3}\right]$ and $(\mathbf{e}, \mathbf{f})$ are for rainy conditions, where $\mathrm{R}$ is the rainfall rate in $[\mathrm{mm} / \mathrm{h}]$. 
Substituting Equations (7) and (8) into Equation (6), the transfer function of the atmospheric medium can be presented as:

$$
H(j f)=\sqrt{T_{F S}(d)} e^{-[\alpha(f)+j \beta(f)] d}
$$

where $\alpha(f)$ is the attenuation factor, given in terms of the imaginary part of the atmospheric refractivity:

$$
\alpha(f)=\frac{2 \pi f}{c} N^{\prime \prime}(f) \times 10^{-6}
$$

where $\beta(f)$ is the wavenumber of the propagating field, expressed in terms of the real part of the atmospheric refractivity:

$$
\beta(f)=\frac{2 \pi f}{c}+\underbrace{\frac{2 \pi f}{c}\left[N_{0}+N^{\prime}(f)\right] \times 10^{-6}}_{\Delta \beta(f)}
$$

The term $\Delta \beta(f)$ is defined as the incremental phase dispersion caused by the dielectric substances composing the air.

The frequency dependent attenuation and incremental phase dispersion are illustrated in Figure 2, for different weather conditions characterized by relative humidity $R H$ [\%], suspended liquid water content $W\left[\mathrm{~g} / \mathrm{m}^{3}\right]$, and rainfall rate $R[\mathrm{~mm} / \mathrm{h}]$.

The results reveal absorption peaks at 22, 183, and $325 \mathrm{GHz}$, where resonance absorption of water $\left(\mathrm{H}_{2} \mathrm{O}\right)$ occurs, as well as absorption peaks at 60 and $119 \mathrm{GHz}$, due to absorption resonances of oxygen $\left(\mathrm{O}_{2}\right)$. Between these frequencies, minimum attenuation is obtained at $35 \mathrm{GHz}$ (Ka-band), $94 \mathrm{GHz}$ (W-band), $130 \mathrm{GHz}$ (D-band), and $220 \mathrm{GHz}$, which are known as atmospheric transmission "windows". Increasing the water content in the air in different forms (vapor (humidity), suspended water droplets (mist and fog), and drops (rain)) significantly increases the attenuation and incremental phase dispersion.

The model was tested experimentally for various foggy conditions in a controlled environment demonstrating good agreement with measured results [9-11].

\section{Transmission of OFDM Waveform in a Dispersive Medium}

The orthogonal frequency division multiplexing (OFDM) modulation technique is based on a digital multi-carrier signaling. A set of symbols are mapped onto adjacent sub-carriers in the frequency domain, employing an Inverse Fast Fourier Transformation [15]. A Cyclic Prefix (CP) is added to preserve orthogonality and reduce Inter-Symbol Interference (ISI) [16]. One of the main benefits of CP-OFDM is its simplicity, and its susceptibility against ISI and fading.

One of the notable drawbacks of OFDM is its wide bandwidth. While preventing ISI, the use of a Cyclic Prefix leads to a reduction in spectral efficiency, as sending a redundant information. The use of CP-OFDM is also characterized by having high Out of Band Power, leading to possible Adjacent Channel Interference [17]. The transmitted complex envelope of the OFDM signaling is given by [7]:

$$
\widetilde{A}_{\text {in }}(t)=\sum_{k=0}^{K-1}\left[I_{m}(k)+j Q_{m}(k)\right] e^{j k \frac{2 \pi}{T} t}
$$

where $K$ is the number of subcarriers, $I_{m}(k)$ and $Q_{m}(k)$ are the respective in-phase and quadrature components of the $m$ th Quadrature Amplitude Modulation (QAM) symbol transmitted in subcarrier $k$, and $T$ is symbol temporal duration. The Fourier transform of the transmitted complex envelope is:

$$
\widetilde{A}_{i n}(j f)=\sum_{k=0}^{K-1}\left[I_{m}(k)+j Q_{m}(k)\right] \delta\left(f-\frac{k}{T}\right)
$$


Substitution of Equation (12) into Equation (5) results in the received complex envelope:

$$
\widetilde{A}_{\text {out }}(t)={\sqrt{T_{F S}(d)}}^{K-1}\left[I_{m}(k)+j Q_{m}(k)\right] \cdot H\left(\frac{k}{T}+f_{c}\right) \cdot e^{j k \frac{2 \pi}{T} t}
$$

Examination of Equation (14) reveals that the constellation of the received signal is affected by the frequency response $H(j f)$ of the medium. In the case of a wireless link operating in millimeter waves, the resulted complex envelope is affected by the dispersion of both attenuation and phase of the atmospheric refractivity:

$$
\widetilde{A}_{\text {out }}(t)=\sqrt{T_{F S}(d)} \sum_{k=0}^{K-1}\left[I_{m}(k)+j Q_{m}(k)\right] \cdot e^{-\left[\alpha\left(\frac{k}{T}+f_{0}\right)+j \beta\left(\frac{k}{T}+f_{0}\right)\right] d} \cdot e^{j k \frac{2 \pi}{T} t}
$$

Inspection of Equation (15) for the complex envelope reveals the influences of absorptive and dispersive atmospheric propagation effects on the constellation expected at the receiver site. It is noted that not only is attenuation resulted due to the atmospheric absorption, but also a frequency dependent phase shift that leads to a distorted constellation. The expression will be used to study the role of atmospheric refractivity in the determination of the performance of OFDM wireless link operating in millimeter wavelengths. In the following sections, we will focus on EHF bands allocated for the next generation of cellular networks- the 5G.

\section{Technical Specifications of the Next Generation Cellular Network}

The definition of signal frame structure for the next generation cellular network was discussed in detail during the 3GPP and in many regulation forums [18-21]. Requirements throughout the RF specifications defined separately for different frequency ranges (FR). Two different frequency bands are available for the 5G technology, designated as: FR1-410 MHz to 7.125 GHz and FR2-24.25 GHz to $52.3 \mathrm{GHz}[19,21,22]$.

The 5G New Radio (NR) Cellular Network is designed to operate in the FR2 operating bands presented in Table 1. Both Uplink (UL) and Downlink (DL) bands operated in Time Division Duplexing (TDD).

Table 1. Frequency operating bands of New Radio (NR) in Ka-Band spectrum [21,22].

\begin{tabular}{cccc}
\hline Operating Band & Carrier Frequency [GHz] & $\begin{array}{c}\text { Uplink (UL) and } \\
\text { Downlink (DL) } \\
\text { Operating Bands [GHz] }\end{array}$ & Duplex Mode \\
\hline $\mathrm{n} 257$ & 28 & $26.5-29.5$ & $\begin{array}{c}\text { Time Division } \\
\text { Duplexing (TDD) }\end{array}$ \\
\hline $\mathrm{n} 258$ & 26 & $24.25-27.5$ & TDD \\
\hline $\mathrm{n} 260$ & 39 & $37-40$ & TDD \\
\hline $\mathrm{n} 261$ & 28 & $27.5-28.35$ & TDD \\
\hline
\end{tabular}

In addition to FR1 and FR2, the Multiple Gigabit Wireless System (MGWS) was defined to operate in frequencies around $60 \mathrm{GHz}$. The MGWS network can be used in short-range, line-of-sight, and non-line-of-sight circumstances with traditional Wireless Local Area Network (WLAN) topologies [23].

Flexible Multi-Numerology are supported in an effort to present diverse services and user requirements of NR [24]. This numerology is characterized by different values of OFDM waveform parameters, such as SCS (Subcarrier Spacing) and CP (Cyclic Prefix) overhead. According to the 3GPP [22], the maximum channel bandwidth per NR carrier is $400 \mathrm{MHz}$ and the maximum SCS is $480 \mathrm{kHz}$. At least for single numerology cases, the maximum number of subcarriers per band is 
3300 or 6600 [25]. Frame Length is fixed to be $10 \mathrm{~ms}$. The number of OFDM symbols per slot time is depending on subcarrier spacing. For subcarrier spacing less than $60 \mathrm{kHz}$, the number of OFDM symbols per slot is 7 or 14 . For subcarrier spacing greater than $60 \mathrm{kHz}$, the number of OFDM symbols per slot is 14 [26]. Table 2. presents the OFDM parameters designated for the 5G technology.

Table 2. Orthogonal frequency division multiplexing (OFDM) symbols configuration in 5G NR [26].

\begin{tabular}{ccccc}
\hline $\begin{array}{c}\text { Subcarrier Spacing (SCS) } \\
\text { Configuration } \mu\end{array}$ & SCS $\Delta f=2^{\mu} \cdot 15[\mathrm{kHz}]$ & $\begin{array}{c}\text { Number } \\
\text { of Symbols Per } \\
\text { Slot }\end{array}$ & $\begin{array}{c}\text { Number of Slots } \\
\text { Per Frame }\end{array}$ & $\begin{array}{c}\text { Number of OFDM } \\
\text { Symbols Per Subframe }\end{array}$ \\
\hline 0 & 15 & 14 & 10 & 1 \\
\hline 1 & 30 & 14 & 20 & 2 \\
\hline 2 & 60 & 14 & 40 & 4 \\
\hline 3 & 120 & 14 & 80 & 16 \\
\hline 4 & 240 & 14 & 160 & 8 \\
\hline
\end{tabular}

\section{Channel Simulation Model}

The channel model was simulated by integrating the OFDM signaling with the medium frequency dependent transfer function resulted from the millimeter propagation model (MPM) for different weather condition scenarios. The received signal is decoded in the presence of AWGN by the OFDM demodulator.

The flow chart shown in Figure 3 summarizes the procedure employed in the numerical simulation calculating the resulted atmospheric absorptive and dispersive effects on the waveform constellation. Information related to the signaling designated for the next generation cellular network was taken from technical specifications presented above.

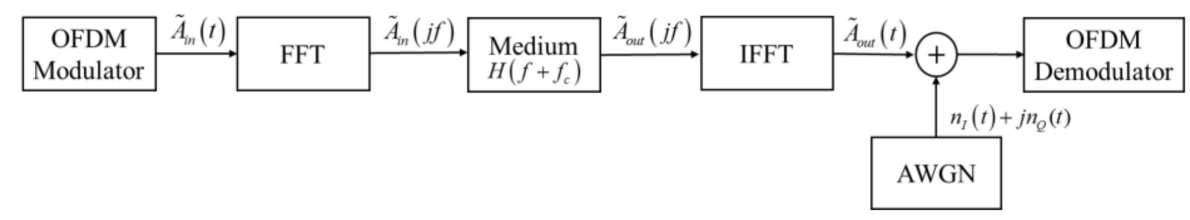

Figure 3. Flow chart of a baseband OFDM complex envelope evolution along the channel.

The model was implemented in MATLAB environment using 5G modeling Toolbox [12]. The model consists of the following steps:

1. Transmitted data signal settings: generation of transmission vector containing a given number $\left(N_{b}=24,000\right.$ or 50,400$)$ of random bits in time domain.

2. Channel properties: the channel characteristics were calculated from the MPM by setting the carrier frequency $(28,39$ or $60 \mathrm{GHz})$ and bandwidths (200 or $400 \mathrm{MHz})$.

3. Signaling: the modulation method for the current analysis is considered to be OFDM with the parameters presented in Table 3: Symbol Rate, Cyclic Prefix Length, Guard Band Length, and Modulation Order. The modulation was built by creating complex QAM envelopes for each of the OFDM subcarriers. The modulator transmits a complex envelop waveform in accordance with Equation (12).

4. The medium: the atmospheric medium was modeled by the MPM to generate the transfer function (9) in the frequency domain for a given distance $(200 \mathrm{~m})$ and different weather condition scenarios. The meteorological parameters were set to: temperature $\left(25^{\circ} \mathrm{C}\right)$, pressure $(1 \mathrm{~atm})$, relative humidity $(R H=0,10,50,100 \%)$ suspended liquid water content $\left(W=0,0.1,1,5 \mathrm{~g} / \mathrm{m}^{3}\right)$, or rain rate $(R=0,5,10,15 \mathrm{~mm} / \mathrm{h})$. Note that for the case of suspended liquid water droplets and rain the relative humidity is set to be $100 \%$. 
5. Noise: Additive White Gaussian Noise (AWGN) with a given Signal-to-noise ratio (SNR) was introduced to the signal.

6. Demodulator: after passing through the atmospheric medium, the received signal is demodulated to decode the digital data.

Table 3. OFDM parameters for the simulation.

\begin{tabular}{ccc}
\hline Parameter & Units & Value \\
\hline Channel Bandwidth & {$[\mathrm{MHz}]$} & 200 \\
\hline Number of Subcarriers & - & 3300 \\
\hline OFDM Subcarrier Spacing & {$[\mathrm{kHz}]$} & 60 \\
\hline Subcarrier Spacing & {$[\mathrm{kHz}]$} & 15 \\
\hline Quadrature Amplitude Modulation (QAM) Modulation Order & - & 6 \\
\hline OFDM Symbols Rate & {$[\mathrm{kSymbols} / \mathrm{sec}]$} & 14 \\
\hline Bits Per Subcarrier & - & 8 \\
\hline Cyclic Prefix Length & - & 64 \\
\hline
\end{tabular}

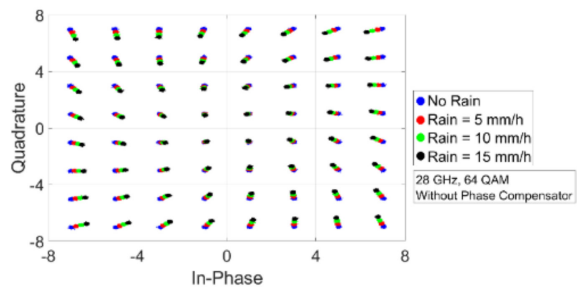

(a)

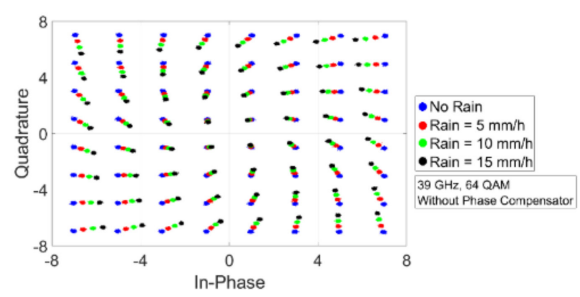

(c)

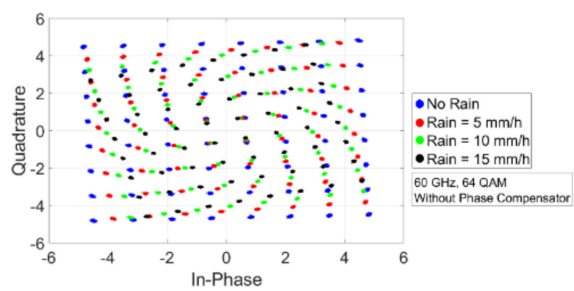

(e)

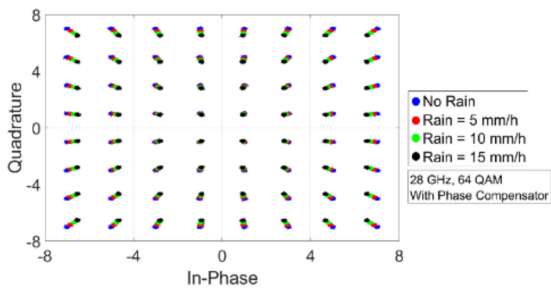

(b)

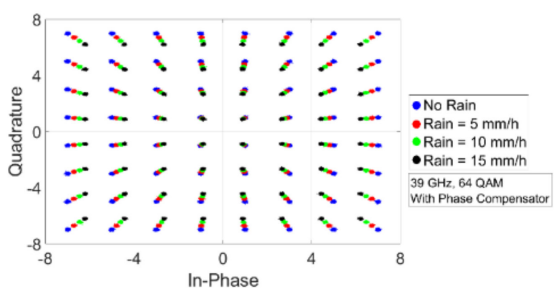

(d)

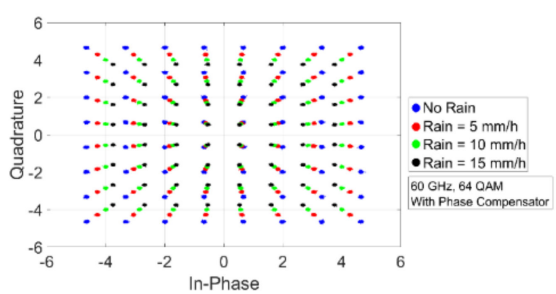

(f)

Figure 4. Simulation results of 64 QAM constellation diagram and effect of Phase Compensator on it at carrier frequencies: (a,b) 28, (c,d) 39, (e,f) $60 \mathrm{GHz}$ for $200 \mathrm{~m}$ wireless link in different rainfall rate. 
The channel performance was studied to find the constellation diagram at the receiver and the resulted Bit Error Rate (BER). The BER is calculated by comparing the received bit sequence with the transmitted one.

The quality of the reception is also measured via the constellation diagram in the signal vector I/Q space. Each point in the constellation diagram represents a given QAM symbol as a vector. The constellation diagram demonstrates amplitude and phase distortions emerging due to the dispersive propagation as well as the additive noise associating with the demodulation.

\section{Simulation Results}

The performance of the OFDM wireless link under various weather conditions was studied using the developed model. A single transmitter and receiver (SISO) transmission link was simulated, within bandwidth of $200 \mathrm{MHz}$, centered at $28 \mathrm{GHz}, 39 \mathrm{GHz}$, or $60 \mathrm{GHz}$. Typical transmission distance of $200 \mathrm{~m}$ is assumed. Simulations were carried out for reference conditions: an ambient temperature of $25^{\circ} \mathrm{C}$ and air pressure of $101 \mathrm{kPa}$ for an urban environment. Using meteorological parameters, the MPM predicts frequency-dependent absorptive and dispersive phenomena, causing distortions in amplitude and phase of propagated waveform.

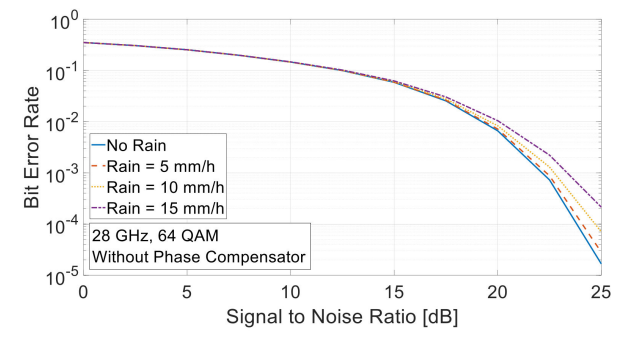

(a)

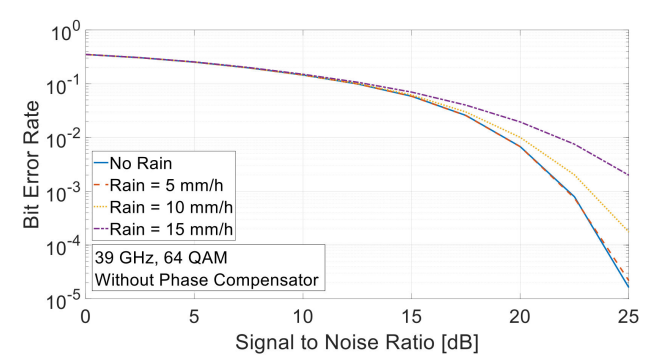

(c)

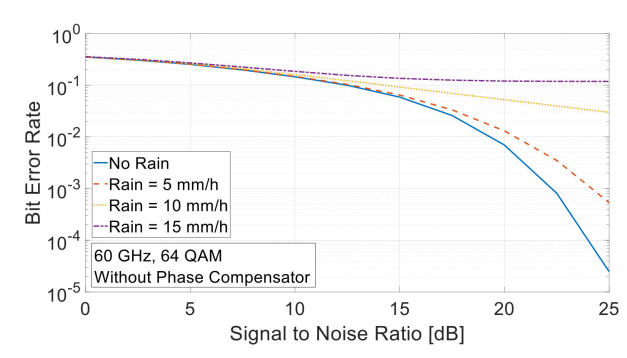

(e)

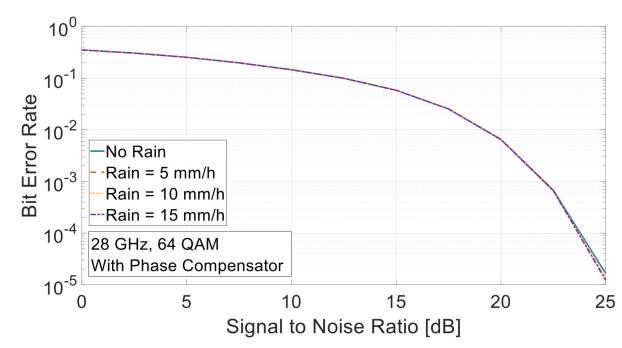

(b)

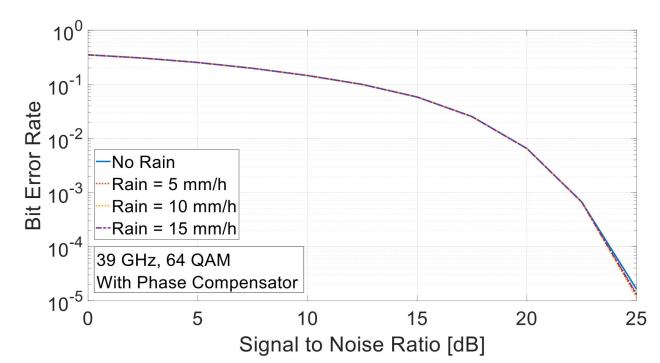

(d)

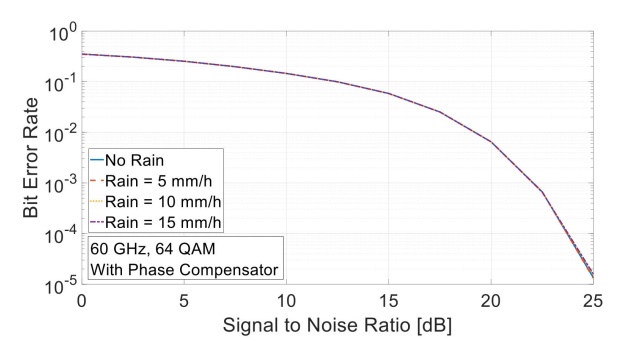

(f)

Figure 5. Bit Error Rate (BER) Performance of 64 QAM and effect of Phase Distortion Compensator on it at carrier frequencies of $(\mathbf{a}, \mathbf{b}) 28,(\mathbf{c}, \mathbf{d}) 39$, and (e,f) $60 \mathrm{GHz}$ for $200 \mathrm{~m}$ wireless link in different rainfall rate. 
In the current work, the presented results are focused on the effect of rain, where the most significant effect on the link budget was identified. The propagation parameters calculated by the MPM model are summarized in Table 3 for the designated 5G bands. The attenuation and phase shift are revealed to increase with the rain rate and with the carrier frequency. These results correspond with experimental climatic wind tunnel results [4].

At the second stage, the effect of the rain intensity on the quality of waveform reception and OFDM demodulation is examined. The bit error ratio (BER) as function of SNR at carrier frequencies 28, 39, and $60 \mathrm{GHz}$ of a wireless link of $200 \mathrm{~m}$ is presented in Figure $4 \mathrm{a}, \mathrm{c}, \mathrm{e}$ for 64-QAM constellation. For each frequency, the BER is found for different rainy scenarios: no rain, 5, 10, and $15 \mathrm{~mm} / \mathrm{h}$. It is important to note that the rain plays a different role at different frequency bands. For instance, setting the carrier frequency to $28 \mathrm{GHz}, \mathrm{SNR}=25 \mathrm{~dB}$, the resulted BER is increased from $2 \times 10^{-5}$ (no rain) to $2 \times 10^{-4}$ in rainy conditions (when the rain intensity is $\mathrm{R}=15 \mathrm{~mm} / \mathrm{h}$ ). However, for $39 \mathrm{GHz}$ carrier frequency, $\mathrm{SNR}=25 \mathrm{~dB}$ and rain intensity of $\mathrm{R}=15 \mathrm{~mm} / \mathrm{h}$, the BER further increases to $2 \times 10^{-3}$ (see Figure $5 \mathrm{a}, \mathrm{c}$ ).

The received signal quality is analyzed by displaying the constellation diagram for each scenario. A contraction of the constellation diagram demonstrates attenuation of the OFDM signal, while rotation of the diagram is a result of phase shift. Figure $4 \mathrm{a}, \mathrm{c}, \mathrm{e}$ shows the effect of rain on 64 QAM constellation diagram at carrier frequencies of 28,39 , and $60 \mathrm{GHz}$ of wireless link of $200 \mathrm{~m}$. The attenuation expressed by $\alpha(f)$ causes the constellation to "shrink". The revealed counterclockwise rotation of the diagram is a result of dispersion of the propagating OFDM signal due to the rain. The rotation angle increases with frequency corresponding to the incremental phase dispersion, given in term of $\Delta \beta(f)$ in Equation (11). Both effects cause the symbols to change their position in the constellation I/Q domain resulting in misinterpretation by the demodulators and erroneous symbol receptions.

While constellation diagrams are useful for graphically visualizing signal data, it is also useful to quantify the disparity between received signal waveform and the ideal constellation by calculating the error vector magnitude (EVM). EVM is the scalar distance between the end points of the measured and ideal phasors and is a measure of how well a digital communications system is performing [27]. As is presented in Figure 6, the higher the QAM signaling order is, the resulted constellation rotation angle (Table 4) at the receiver is more expressed. Therefore, the EVM will increase for high modulation orders, resulting in erroneous detected symbols even at relatively low rainfall intensities.

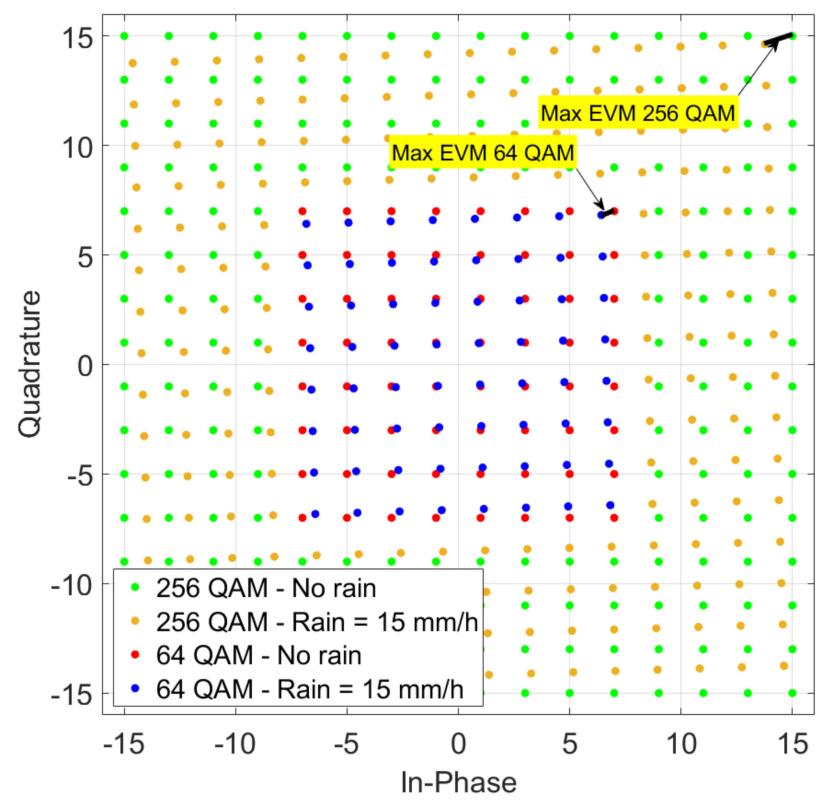

Figure 6. Comparison of 64 QAM and 256 QAM constellation diagrams at a carrier frequency of $28 \mathrm{GHz}$ for $200 \mathrm{~m}$ wireless link for different rainfall rates. 
Table 4. Propagation factors of 28,39 , and $60 \mathrm{GHz}$ for $200 \mathrm{~m}$ wireless link.

\begin{tabular}{|c|c|c|c|c|c|}
\hline Parameter & Units & No Rain & $5 \mathrm{~mm} / \mathrm{h}$ & $10 \mathrm{~mm} / \mathrm{h}$ & $15 \mathrm{~mm} / \mathrm{h}$ \\
\hline \multicolumn{6}{|c|}{$28 \mathrm{GHz}$} \\
\hline Attenuation Factor & {$[\mathrm{dB} / \mathrm{km}]$} & 0.3074 & 1.0460 & 1.8501 & 2.6809 \\
\hline Phase Factor & {$[\mathrm{deg} / \mathrm{km}]$} & 1.7740 & 3.9402 & 6.5050 & 9.5047 \\
\hline Attenuation for $200 \mathrm{~m}$ & {$[\mathrm{~dB}]$} & 0.0615 & 0.2092 & 0.3700 & 0.5362 \\
\hline Incremental part of phase for $200 \mathrm{~m}$ & [deg] & 0.3548 & 0.7880 & 1.3010 & 1.9009 \\
\hline \multicolumn{6}{|c|}{$39 \mathrm{GHz}$} \\
\hline Attenuation Factor & {$[\mathrm{dB} / \mathrm{km}]$} & 0.3310 & 1.7524 & 3.1170 & 4.4610 \\
\hline Phase Factor & {$[\mathrm{deg} / \mathrm{km}]$} & 2.9819 & 2.6178 & 9.0821 & 16.4558 \\
\hline Attenuation for $200 \mathrm{~m}$ & [dB] & 0.0662 & 0.3505 & 0.6234 & 0.8922 \\
\hline Incremental part of phase for $200 \mathrm{~m}$ & [deg] & 0.5964 & 0.5236 & 1.8164 & 3.2912 \\
\hline \multicolumn{6}{|c|}{$60 \mathrm{GHz}$} \\
\hline Attenuation Factor & {$[\mathrm{dB} / \mathrm{km}]$} & 14.63 & 17.5066 & 19.8636 & 22.0577 \\
\hline Phase Factor & {$[\mathrm{deg} / \mathrm{km}]$} & 3.3368 & 11.9821 & 28.9276 & 47.4929 \\
\hline Attenuation for $200 \mathrm{~m}$ & {$[\mathrm{~dB}]$} & 2.926 & 3.5013 & 3.9727 & 4.4115 \\
\hline Incremental part of phase for $200 \mathrm{~m}$ & [deg] & 0.6674 & 2.3964 & 5.7855 & 9.4986 \\
\hline
\end{tabular}

\section{Channel Performance Improvement via Phase Distortion Compensation}

As mentioned in Section 3, the effect of the atmosphere on the communication channel is expressed by the attenuation coefficient $\alpha(f)$ and by the incremental, frequency dependent phase dispersion $\Delta \beta(f)$, given in Equations (10) and (11), respectively. Figure 4a,c,e presents the effect of these two parameters on the modulated signal, revealing respective "shrink" and "rotation" in the constellation. While the effect of the attenuation $\alpha(f)$ is usually considered in an appropriate design of the link budget, we suggest to neutralize the phase dispersion $\Delta \beta(f)$ caused by the atmospheric medium by adding a phase compensator in the pre-demodulating stage in the receiver MMW chain (see Figure 7). The frequency dependent phase dispersion coefficient can be estimated by meteorological conditions at the receiver site, including relative humidity $R H[\%]$, suspended liquid water content $W\left[\mathrm{~g} / \mathrm{m}^{3}\right]$, and rainfall rate $R[\mathrm{~mm} / \mathrm{h}]$.

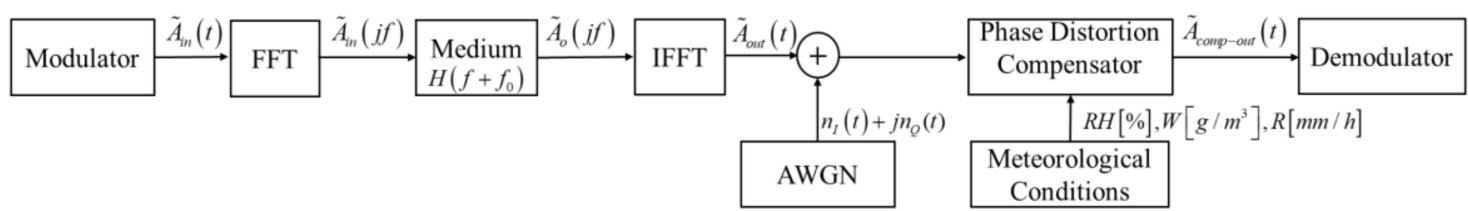

Figure 7. Flow chart of millimeter wave (MMW) link including Phase Distortion Compensator in the receiver site.

Figure $4 b, d$, $f$ shows a positive effect of the Phase Compensator on a distorted 64 QAM constellation diagram for different rainy scenarios: no rain, 5, 10, and $15 \mathrm{~mm} / \mathrm{h}$. As it presented, Phase Distortion Compensator completely neutralizes the "rotation" effect dramatically. As a result, the receiver demodulator becomes able to reproduce the data almost as in no-rain scenarios (see Figure $5 b, d, f$ ).

Table 5 summarizes the expected performances of the average EVM and BER when an adaptive phase compensator is installed before the OFDM demodulation. A comparison with the performances attained when no compensating mechanism is applied during rainy conditions demonstrates that the proposed novel approach improves the reliability of the channel, leading to a reduction in the EVM 
and consequently lowering the error rate. A quantitative measure of the improvement is expressed via the average $E V M$ relative reduction (in [\%]):

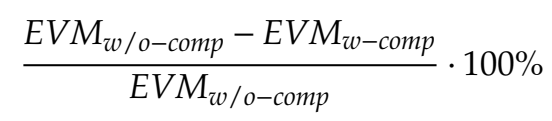

Table 5. Average EVM and BER improvements due to Phase Dispersion Compensation for $200 \mathrm{~m}$ wireless link operating with 64QAM and Signal-to-noise ratio $(\mathrm{SNR})=25 \mathrm{~dB}$.

\begin{tabular}{|c|c|c|c|c|c|c|}
\hline \multirow{2}{*}{$\begin{array}{c}\text { Frequency } \\
\text { [GHz] }\end{array}$} & \multirow{2}{*}{$\begin{array}{c}\text { Rainfall } \\
\text { Rate }[\mathrm{mm} / \mathrm{h}]\end{array}$} & \multicolumn{2}{|c|}{ Without Compensator } & \multicolumn{2}{|c|}{ With Compensator } & \multirow{2}{*}{$\begin{array}{l}\text { Improvement } \\
\text { Factor [\%] }\end{array}$} \\
\hline & & $\begin{array}{l}\text { Average EVM } \\
\text { EVM }_{w / o-c o m p}\end{array}$ & BER & $\begin{array}{l}\text { Average EVM } \\
E V M_{w-c o m p}\end{array}$ & BER & \\
\hline \multirow{3}{*}{28} & 5 & 0.170 & $2.817 \times 10^{-5}$ & 0.165 & $1.210 \times 10^{-5}$ & 2.99 \\
\hline & 10 & 0.364 & $7.044 \times 10^{-5}$ & 0.335 & $1.548 \times 10^{-5}$ & 7.85 \\
\hline & 15 & 0.567 & $2.137 \times 10^{-4}$ & 0.507 & $1.667 \times 10^{-5}$ & 10.61 \\
\hline \multirow{3}{*}{39} & 5 & 0.346 & $2.183 \times 10^{-5}$ & 0.301 & $1.151 \times 10^{-5}$ & 12.79 \\
\hline & 10 & 0.687 & $1.756 \times 10^{-4}$ & 0.573 & $1.290 \times 10^{-5}$ & 16.58 \\
\hline & 15 & 1.028 & $1.996 \times 10^{-3}$ & 0.839 & $1.369 \times 10^{-5}$ & 18.39 \\
\hline \multirow{3}{*}{60} & 5 & 0.514 & $5.335 \times 10^{-4}$ & 0.397 & $1.349 \times 10^{-5}$ & 22.69 \\
\hline & 10 & 0.953 & $2.994 \times 10^{-2}$ & 0.694 & $1.528 \times 10^{-5}$ & 27.19 \\
\hline & 15 & 1.376 & $1.183 \times 10^{-1}$ & 0.961 & $1.587 \times 10^{-5}$ & 30.11 \\
\hline
\end{tabular}

\section{Conclusions}

The transmission quality of the 5th generation (5G) millimeter wave (MMW) wireless communication was studied for various atmospheric weather conditions. The current work is focused on the investigation of the attenuation and phase shift introduced by weather effects, especially the influence of different rainy scenarios.

The link reliability was examined via constellation diagram and BER in the presence of AWGN for different rainfall rates. Simulation results indicate that OFDM channel performance is strongly affected by rainy conditions. It is shown that absorptive and dispersive behavior of the medium transfer function results in distorted magnitude and phase QAM signaling, leading to an increased error rate.

The OFDM MMW channel performance can be significantly improved by considering the effects emerging due to local atmospheric conditions in the receiver demodulator scheme. Online weather reports from local meteorological stations can provide side information on the propagation characteristics that will enable compensation of waveform magnitude and phase distortions at the receiver. This will significantly reduce the error rate leading to an improvement of the link availability and reliability. Furthermore, the on-line meteorological data could also be useful for selecting the optimal parameters for the QAM constellation and the appropriate OFDM modulation numerology to increase network efficiency.

It is demonstrated that by employing an adaptive, pre-demodulation phase correction scheme that the atmospheric dispersion phenomena on the millimeter wave can be compensated, resulting in an improvement of the link reliability, as summarized in Table 5.

It is important to note that by using Phase Dispersion Compensator in the pre-demodulation stage of the receiver, the reliability of the channel during rainy conditions is improved. It is shown that for all examined scenarios, even for extremely bad weather conditions, the compensation revealed a reduction in the error probability, almost to the level expected in clear weather (no rain) scenarios (see Figure 5). 
The necessity of phase compensation depends on the link operational frequency, the signal to noise ratio, as well as on the rain intensity. Major improvements are demonstrated in high rain rates, when the weather conditions cause severe phase distortions. It is also shown that for frequency bands, where highly absorptive and dispersive atmospheric effects are expected (as in $60 \mathrm{GHz}$ ), the compensator modify the phase distortions and as a result reduced EVM and lower error probability.

Author Contributions: Conceptualization, Y.G., G.A.P. and Y.P.; Data curation, Y.G.; Formal analysis, Y.G., G.A.P. and Y.P.; Investigation, Y.G., G.A.P. and Y.P.; Methodology, G.A.P. and Y.P.; Project administration, Y.P.; Software, Y.G. and G.A.P.; Supervision, G.A.P. and Y.P.; Validation, Y.G.; Visualization, Y.G.; Writing-original draft, Y.G.; Writing - review \& editing, G.A.P. and Y.P. All authors have read and agreed to the published version of the manuscript.

Funding: This research received no external funding.

Conflicts of Interest: The authors declare no conflict of interest.

\section{References}

1. Rappaport, T.S.; Xing, Y.; MacCartney, G.R.; Molisch, A.F.; Mellios, E.; Zhang, J. Overview of Millimeter Wave Communications for Fifth-Generation (5G) Wireless Networks-With a Focus on Propagation Models. IEEE Trans. Antennas Propag. 2017, 65, 6213-6230. [CrossRef]

2. Federal Communications Commission. Fact Sheet: Spectrum Frontiers Proposal to Identify, Open Up Vast Amounts of New High-Band Spectrum for Next Generation (5G) Wireless Broadband. Available online: https://www.fcc.gov/document/fact-sheet-spectrum-frontiers-item (accessed on 7 June 2020).

3. Lathi, B.P.; Ding, Z. Modern Digital and Analog Communication Systems, 5th ed.; Oxford University Press: New York City, NY, USA, 2019.

4. Hilt, A. Availability and Fade Margin Calculations for 5G Microwave and Millimeter-Wave Anyhaul Links. Appl. Sci. 2019, 9, 5240. [CrossRef]

5. Antes, J.; Boes, F.; Messinger, T.; Lewark, U.J.; Mahler, T.; Tessmann, A.; Henneberger, R.; Zwick, T.; Kallfass, I. Multi-Gigabit Millimeter-Wave Wireless Communication in Realistic Transmission Environments. IEEE Trans. Terahertz Sci. Technol. 2015, 5, 1078-1087. [CrossRef]

6. Matricciani, E. A Relationship Between Phase Delay and Attenuation Due to Rain and Its Applications to Satellite and Deep-Space Tracking. IEEE Trans. Antennas Propag. 2009, 57, 3602-3611. [CrossRef]

7. Pinhasi, Y.; Yahalom, A.; Harpaz, O.; Vilner, G. Study of ultra-wideband transmission in the extremely high frequency (EHF) band. IEEE Trans. Antennas Propag. 2004, 52, 2833-2842. [CrossRef]

8. Pinhasi, Y.; Yahalom, A.; Pinhasi, G.A.; Lutvak, M. Ultra Wideband Wireless Communications in the $60 \mathrm{GHz}$ Band. WSEAS Transact. Comm. 2010, 12, 773-781.

9. Golovachev, Y.; Etinger, A.; Pinhasi, G.A.; Pinhasi, Y. Millimeter Wave High Resolution Radar Accuracy in Fog Conditions-Theory and Experimental Verification. Sensors 2018, 18, 2148. [CrossRef] [PubMed]

10. Golovachev, Y.; Etinger, A.; Pinhasi, G.A.; Pinhasi, Y. Propagation properties of sub-millimeter waves in foggy conditions. J. Appl. Phys. 2019, 125, 151612. [CrossRef]

11. Etinger, A.; Golovachev, Y.; Shoshanim, O.; Pinhasi, G.A.; Pinhasi, Y. Experimental Study of Fog and Suspended Water Effects on the 5G Millimeter Wave Communication Channel. Electronics 2020, 9, 720. [CrossRef]

12. 5G Toolbox MATLAB, MathWorks Inc. Available online: https://www.mathworks.com/products/5g.html (accessed on 7 June 2020).

13. Liebe, H.J. MPM? An atmospheric millimeter-wave propagation model. Int. J. Infrared Millim. Waves 1989, 10, 631-650. [CrossRef]

14. Liebe, H.J.; Hufford, G.A.; Manabe, T. A model for the complex permittivity of water at frequencies below 1 THz. Int. J. Infrared Millim. Waves 1991, 12, 659-675. [CrossRef]

15. Gerzaguet, R.; Bartzoudis, N.G.; Baltar, L.G.; Berg, V.; Dore, J.-B.; Kténas, D.; Font-Bach, O.; Mestre, X.; Payaró, M.; Färber, M.; et al. The $5 \mathrm{G}$ candidate waveform race: A comparison of complexity and performance. EURASIP J. Wirel. Commun. Netw. 2017, 2017, 1312. [CrossRef] 
16. An, C.; Kim, B.; Ryu, H.-G. Design and evaluation of spectrum efficient WR-OFDM system for 5G and B5G mobile system. In Proceedings of the 2017 IEEE International Conference on Microwaves, Antennas, Communications and Electronic Systems (COMCAS), Tel Aviv, Israel, 13-15 November 2017; pp. 1-5.

17. Levy, D.; Reichman, A. Filter bank multi carrier modulation performance. In Proceedings of the 2017 IEEE International Conference on Microwaves, Antennas, Communications and Electronic Systems (COMCAS), Tel Aviv, Israel, 13-15 November 2017; pp. 1-6.

18. Mourad, A.; Yang, R.; Lehne, P.H.; De La Oliva, A. A Baseline Roadmap for Advanced Wireless Research Beyond 5G. Electronics 2020, 9, 351. [CrossRef]

19. Series, M. IMT Vision-Framework and Overall Objectives of the Future Development of IMT for 2020 and Beyond. In Recommendation ITU; Electronic Publication: Geneva, Switzerland, 2015.

20. Guey, J.-C.; Liao, P.-K.; Chen, Y.-S.; Hsu, A.; Hwang, C.-H.; Lin, G. On 5G radio access architecture and technology [Industry Perspectives]. IEEE Wirel. Commun. 2015, 22, 2-5. [CrossRef]

21. Gozalvez, J. New 3GPP Standard for IoT [Mobile Radio]. IEEE Veh. Technol. Mag. 2016, 11, 14-20. [CrossRef]

22. 3GPP Technical Specification 38.101-2. NR. Available online: https://www.3gpp.org/dynareport/38101-2.htm (accessed on 7 June 2020).

23. ITU-R. M.2003-2, Multiple Gigabit Wireless Systems in frequencies around 60 GHz. Available online: https://www.itu.int/rec/R-REC-M.2003-2-201801-I/en (accessed on 7 June 2020).

24. Yazar, A. Flexible Multi-Numerology Systems for 5G New Radio. J. Mob. Multimed. 2018, 14, 367-394. [CrossRef]

25. 3GPP Technical Report TR 38.802. Available online: https://www.3gpp.org/dynareport/38802.htm (accessed on 7 June 2020).

26. 3GPP Technical Specification TS 38.211. NR. Available online: https://www.3gpp.org/dynareport/38211.htm (accessed on 7 June 2020).

27. Keysight Technologies, Digital Modulation in Communication Systems-An Introduction. Available online: https://iterature.cdn.keysight.com/litweb/pdf/5965-7160E.pdf (accessed on 7 June 2020).

(C) 2020 by the authors. Licensee MDPI, Basel, Switzerland. This article is an open access article distributed under the terms and conditions of the Creative Commons Attribution (CC BY) license (http://creativecommons.org/licenses/by/4.0/). 